\title{
Z-contrast STEM imaging of rare earths segregation at crystal/intergranular film interfaces in $\mathrm{Si}_{3} \mathrm{~N}_{4}$ ceramics
}

\author{
N. Shibata, , G.S. Painter,**, P.F. Becher** and S.J. Pennycook*** \\ * Institute of Enginnering Innovation, The University of Tokyo, Tokyo, Japan \\ ** Metals and Ceramics Division, Oak Ridge National Laboratory, PO Box 2008, Oak Ridge, TN \\ 37831-6064 \\ *** Condensed Matter Sciences Division, Oak Ridge National Laboratory, PO Box 2008, Oak \\ Ridge, TN 37831-6031
}

Tailoring microstructures is very important for obtaining high-performance silicon nitride $\left(\mathrm{Si}_{3} \mathrm{~N}_{4}\right)$ ceramics [1]. Anisotrpic grain growth behavior of $\mathrm{Si}_{3} \mathrm{~N}_{4}$ grains can be used to form very elongated grains, which serve as reinforcing media analogous to fiber reinforced materials. However, the formation of such anisotropic grains is very sensitive to the dopant cations used in the sintering additives [2]. Understanding the underlying atomistic mechanism of these dopant effects is key to design high-performance $\mathrm{Si}_{3} \mathrm{~N}_{4}$ ceramics through microstructure optimization.

Recent observations showed that lanthanum atoms, which dramatically accelerate anisotropic grain growth, preferentially segregate to the prismatic grain surfaces of $\beta-\mathrm{Si}_{3} \mathrm{~N}_{4}$ ceramics [3]. Also, other rare earth dopants are reported to segregate the prismatic grain surfaces [4]. While other rare earths are less effective in promoting anisotropic grain growth, possible differences in segregation and adsorption behaviour of a series of rare earth elements at the atomic level has not been addressed. Here, aberration-corrected Z-contrast STEM is used to observe typical grain boundary films in Lu, Gd and $\mathrm{La}$ doped $\mathrm{Si}_{3} \mathrm{~N}_{4}$ ceramics, which cover the range of stable +3 lanthanide ions that lead to increasing grain growth anisotropy.

Figure 1 shows Z-contrast STEM images of typical grain boundaries in Lu, Gd and La-doped $\mathrm{Si}_{3} \mathrm{~N}_{4}$ ceramics [5]. Each grain on the right side is aligned along the [0001] projection of $\beta-\mathrm{Si}_{3} \mathrm{~N}_{4}$ with the smooth (1010) prismatic plane imaged edge-on. The left hand side grain is oriented with a higher index incident beam direction. The bright spots in the right-hand grains correspond to the Si columns, while the intergranular film (IGF) is the vertically aligned band near the center of each image. The very bright spots in the IGF represent high atomic number atoms (e.g. $Z=71(\mathrm{Lu}), 64(\mathrm{Gd}), 57(\mathrm{La})$, confirmed by energy-dispersive X-ray spectroscopy (EDS) microanalysis). Each periodic bright spot along the edge of the right-hand interface (indicated by the red arrows) corresponds to a concentration of dopant atoms attached to the prism surfaces of grains. In each case, rare earth attachment at the anion terminated prismatic surface forms the first cation layer in the IGF. Figure 2 shows magnified images of the interface between the prismatic grain surface and IGF for each rare earth. Comparison of the interface images reveals that, in these three dopant systems, a distinct sequence of surface occupations is observed by the Z-contrast STEM. The difference in surface occupancies in each dopant system is also confirmed by the first-principles cluster calculations [5]. It is found that the segregation energy of these dopants to the prismatic $\beta-\mathrm{Si}_{3} \mathrm{~N}_{4}$ surface is the key parameter to determine the anisotropic grain growth behavior in doped $\mathrm{Si}_{3} \mathrm{~N}_{4}$ ceramics. Thus, elegant combination of Z-contrast STEM with theory now provides the basis for designing very tough microstructures from the atomistic dimensions. 
References

[1] I-W. Chen et al (ed), MRS proceedings 287 (Mater. Res. Soc. Pittsburgh, PA, 1993).

[2] Hoffmann, M.J., Gu, H \& Cannon, R.M.. Interfacial Engineering for Optimized Properties II 586, 65-74(Mater. Res. Soc. Warrendale, PA, 2000).

[3] N. Shibata et al., Nature, 428,730-33 (2004).

[4] A.Ziegler et al., Science, 306, 1763 (2004).

[5] N. Shibata et al., submitted.

[6] We thank R. L. Satet and M. J. Hoffmann for supplying the silicon nitride ceramics used in this study. This work was supported by the US Department of Energy, Office of Basic Energy Sciences, Division of Materials Sciences and Engineering.

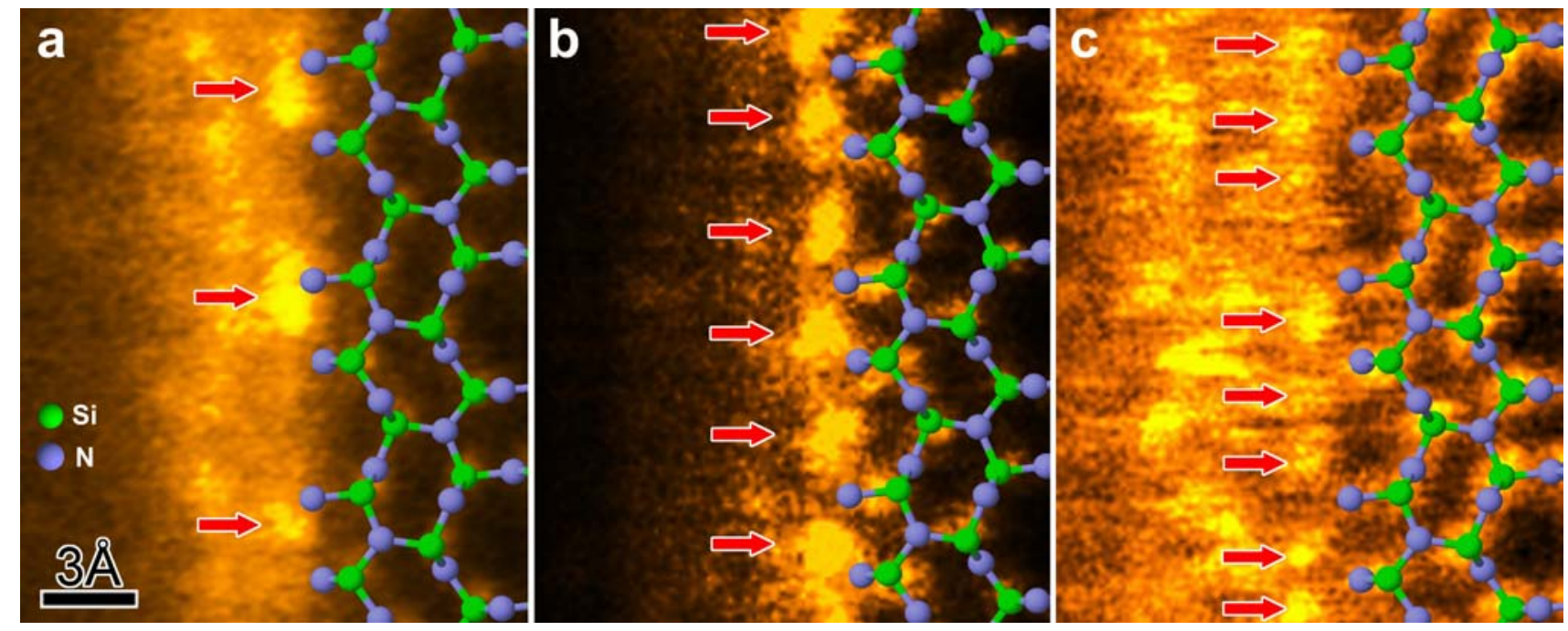

FIG.1: Z-contrast images of the IGF/crystal interfaces in (a) Lu, (b)Gd, and (c) La systems, respectively. Arrows indicate the position of rare earth attachment sites on the prismatic crystal surface.
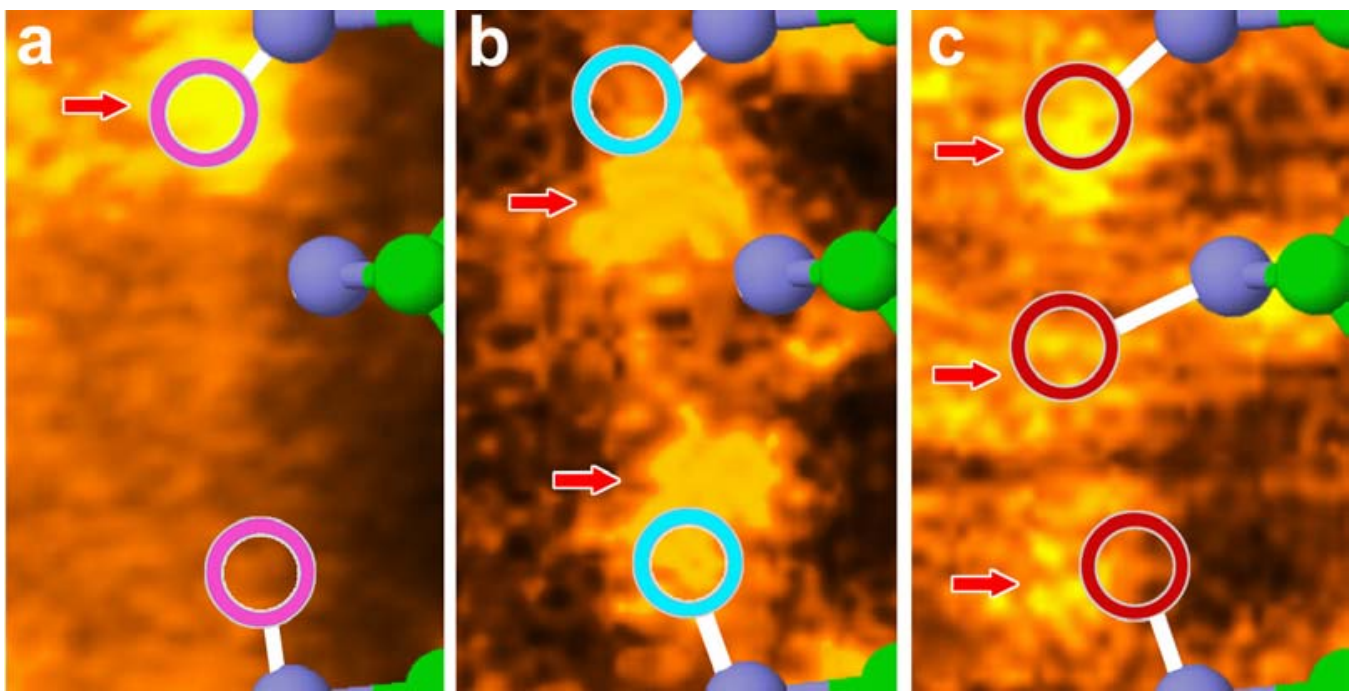

FIG.2: Magnified Z-contrast images of the IGF/crystal interfaces in (a) Lu, (b)Gd, and (c) La systems, respectively. Open circles correspond to the position of stable attachment sites for each dopant predicted by first-principles cluster calculations [5]. 